www.jmscr.igmpublication.org

Impact Factor (SJIF): 6.379

Index Copernicus Value: 79.54

ISSN (e)-2347-176x ISSN (p) 2455-0450

crossrefDOI: https://dx.doi.org/10.18535/jmscr/v6i11.79

\title{
Giant Prostatic Urethral Calculus: A Rare Case Report with Total Endoscopic Management
}

\author{
Authors \\ Dr Venkat A.Gite, Dr Prakash Sankapal, Dr Atul Singal, Dr Mayank Agarwal \\ JJ Hospital Mumbai, India
}

\begin{abstract}
The giant prostatic calculus within the prostatic urethra is a rare clinical entity in the young population. Less than 20 cases have been reported in literature ${ }^{(1)}$.

Most of the calculi in the urethra migrate from the bladder and obliterate the urethra. These stones are often composed of calcium phosphate or calcium oxalate. The decision of treatment strategy is affected by the size, shape and position of the calculus and by the status of the urethra.

We present a case of a 40 year old male with a giant calculus in the prostatic urethra which was successfully managed with an endoscopic procedure. Stone analysis suggestive of calcium oxalate stone.

Keywords: LUTS, Giant, prostatic calculus, PCCL (Per Cutaneous Cystolithotrity).
\end{abstract}

\section{Introduction}

Prostatic urethral calculi are rare in children and in young men below the age of 40 years, but are common in males above 50 years of age $^{(2)}$. Prostatic parenchymal calculi are usually incidental findings on a computed tomography (CT) scan or transrectal ultrasound. They are typically asymptomatic and may be associated with benign prostatic hyperplasia, and prostatic cancer. Prostatic calculus of giant size is very rare in routine clinical practice and literature. Most of them were treated by surgery. We report a case of giant prostatic calculus in a young man treated totally by an endoscopic approach.

\section{Case Report}

A 40 year old man presented with complaints of lower urinary tract symptoms (LUTS). He reported a 1 year history of dysuria and hypo gastric pain with feeling of incomplete emptying. Patient had recurrent urinary tract infection since 1 year. On digital rectal examination, stony hardness was noted through the anterior rectal wall. The rest of the examination was unremarkable. Renal function test was found to be normal. Urine routine and microscopy showed plenty of pus cells and urine culture showed growth of Proteus mirabilis. A kidney, urethra and bladder (KUB) X ray showed a large calculus in the prostatic urethra (Figure 1). Ultrasound abdomen revealed mild bilateral hydroureteronephrosis with thickening of bladder wall and a giant prostatic calculus of size $6.5 \times 4 \mathrm{~cm}$. Ascending cystourethrogram (ASU) and a micturating cystourethrogram (MCU) showed a large calculus in the prostatic urethra with passage of contrast from urethral meatus to bladder and bilateral vesicoureteric reflux (VUR) of grade 3 


\section{JMSCR Vol||06||Issue||11||Page 453-455||November}

(Figure 2). Non contrast computed tomography (NCCT) of KUB showed a hyperdense calculus [Hounsfield unit (HU).647] of size $6.5 \times 3.5 \mathrm{~cm}$ in the bladder neck and prostatic urethra (Figure 3).

After treating the urinary tract infection with culture speific antibiotics, the patient underwent urethrocystoscopy which revealed normal anterior urethra with complete obstruction of the prostatic urethra by a giant calculus. A Percutaneous suprapubic endoscopic approach was used to treat this patient. This giant prostatic urethral calculus (Figure 4) was successfully managed by percutaneous cystolithotripsy (PCCL) using a pneumatic lithotripter and suprapubic catherisation (SPC) was done.

Post operative course was uneventful and patient was discharged on $3^{\text {rd }}$ post op day.

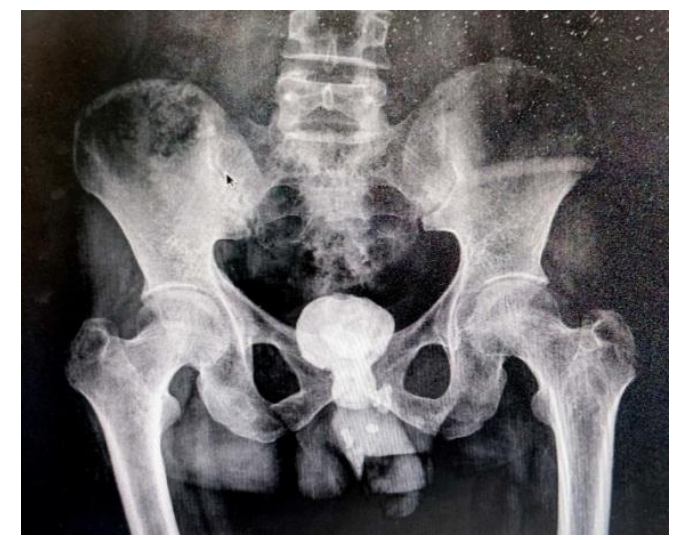

Legend 1 Kdney, urethra and bladder (KUB) X ray showed a large calculus in the prostatic urethra.

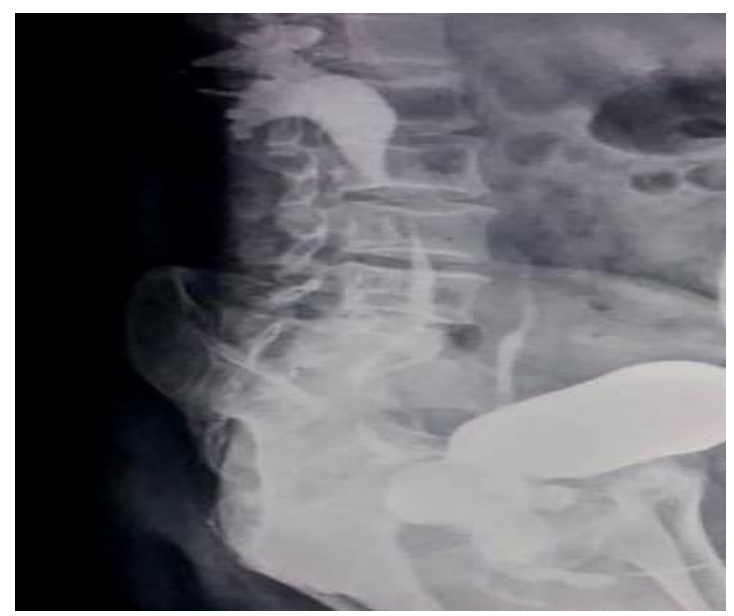

Legend 2-ASUand MCU showed a large calculus in the prostatic urethra with passage of contrast from urethral meatus to bladder and bilateral VUR of grade III.

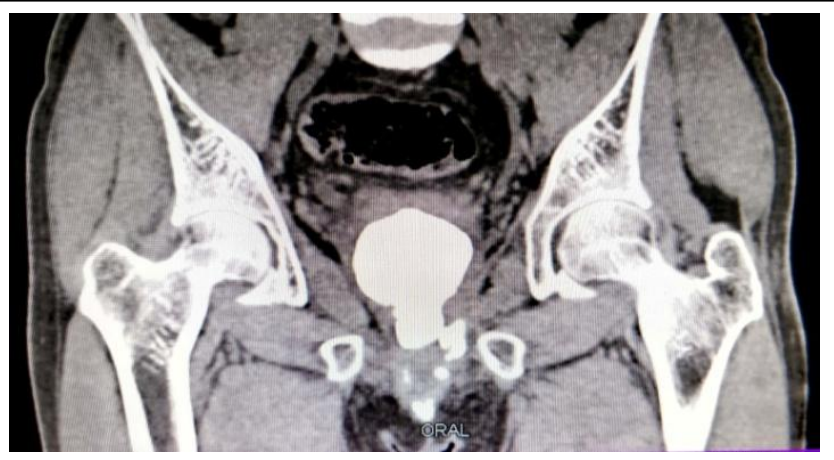

Legend 3 NCCT KUB showed a hyperdense calculus of size $6.5 \times 3.5 \mathrm{~cm}$ in the bladder neck and prostatic urethra

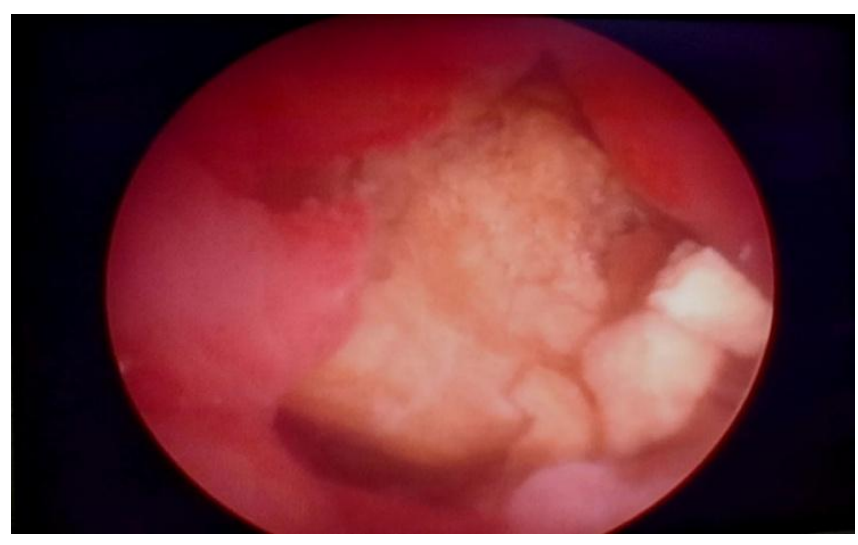

Legend 4 Percutaneous suprapubic endoscopic view of giant prostatic urethral calculus.

\section{Discussion}

Less than 20 cases of giant prostatic calculi have been reported in literature. The etiology of prostatic calculi remains unclear. They can be classified in to endogenous or primary, and exogenous or secondary. Primary native calculi are usually small and multiple and secondary calculi are usually larger ${ }^{(3)}$. Endogenous calculi can be formed from direct precipitation of elements present in prostatic secretion stasis that results in obstruction, inflammation and chronic infection of the prostatic ducts. They generally do not cause acute symptoms because of slow development. When the stone is exogenous, they are formed from constituents of urine, as shown by crystallographic studies of prostatic calculi ${ }^{(4)}$. These secondary stones are much more common and are usually made of calcium oxalate and phosphate. They often cause acute symptoms causing retention, frequency, dysuria, poor stream or dribbling ${ }^{(3)}$. 
The prostatic calculi may be associated with many diseases. These include BPH, adenocarcinoma, genitourinary tuberculosis and chronic infection $^{(5,6)}$.

Patients can present with LUTS, urinary retention, pain, urethral strictures or symptomatic urethral stones. Chronic prostatitis and recurrent urinary tract infection have been implicated in their development ${ }^{(7)}$.

Clinical examination is usually normal but sometimes these calculi are found on digital rectal examination (DRE) as a hard nodule that was suspected more of a prostatic cancer. The diagnosis of prostatic calculus is radiological. Standard radiographs or ultrasound especially prostate transrectal ultrasound which has better diagnostic sensitivity ${ }^{(8)}$. Retrograde cystography is indicated to detect associated urethral diseases.

Most asymptomatic prostatic calculi do not require treatment. Treatment is influenced by the size, shape and position of the calculus and by the status of the urethra $^{(9)}$. If the stone is large and immobile, it may be extracted via a perineal or suprapubic approach. In most cases the calculus was extracted via a transvesical approach and external urethrotomy. Giant prostatic urethral calculi usually treated by open surgical method but in our case it is totally managed by endoscopic $\operatorname{method}^{(3,4)}$. During stone fragmentation from percutaneously we placed another 19Fr sheath from perurethral region. Percutaneous transvesical fragmentation makes disimpaction of prostatic stone while perurethral scope helped in pushing the stone from perurethrally to make fragmentation of stone easy and avoiding the migration of fragmented stones to the urethra

In our case, the calculus was successfully fragmented using a pneumatic lithotripter via percutaneous transvesical approach. The patient was stone free without any intraoperative and postoperative complications.
Conclusion: Giant prostatic urethral calculus is very rare and usually treated by open surgical method.

Total endoscopic management is attractive alternative as it is having less morbidity.

Total endoscopic management should be tried in all cases of prostatic urethral calculi whenever its feasible and possible.

\section{References}

1. Shah SK, Chau MH, Schnepper GD, Lui PD. Open prostatolithotomy for the management of giant prolculuistatic calculi. Urology. 2007;70(5):1008 e 1009=1010.

2. Klimas R, Bennett B, Gardner WA (1985) Prostatic calculus ; a review. Prostate 7:91=6

3. Prabhuswamy VK, Tiwari R, Krishnamoorthy R. A giant dumbbell shaped vesico prostatic urethral calculus: a case report and review of literature. Case Rep Urol. 2013;2013:167635

4. Ramirez CT, Ruiz JA, Gomez et al. Crystallograohic study of prostati calculi. J Urol 1980: 124; 840 843. PubMed /Google Scholar

5. Cambell urology: Urinary lithiasis: etiology, diagnosis, and medical management. 1998. Philadelphia. WB Saunders. Pub Med/Google Scholar

6. Cambell urology: Prostatitis and related disorders. 1998. Philadelphia. WB Saunders. Pub Med/Google Scholar

7. Beusing S, Kilciler M, Akay O, et al. Endoscopic treatment of multiple prostatic calculi causing urinary retention. 2005.Int J Urol. 12: 693,695. Pub Med/Google Scholar

8. Bock E, Calugi V, Stolfi V, Rossi P, D Ascenzo R, Solivetti FM. Calcification of the prostate: a transrectal echographic study. Radiol Med. 1989 May: 77(5);501.3. Pub Med/Google Scholar

9. Non M, Resnick M(2002) Calculi of prostate and seminal vesicles; urethral calculi in men. In: Walsh PC (ed) Campbell, s urology, $8^{\text {th }}$ edn. Saunders Inc.,Philadelphis, pp3287,3289 